\title{
TRANSFERÊNCIA MONETÁRIA NO BRASIL E NO CHILE: COMPARANDO IDEIAS E O PAPEL DE INSTRUMENT CONSTITUENCIES'
}

\author{
Maria Clara Oliveira ${ }^{\mathrm{a}}$ \\ a Instituto de Direito Económico, Financeiro e Fiscal da Faculdade de Direito \\ da Universidade de Lisboa e Centro de Estudos da Metrópole. Lisboa, Portugal. \\ E-mail: mariaclara.g.oliveira@gmail.com \\ Orcid: 0000-0003-3045-6300. \\ Renata Mirandola Bichirb

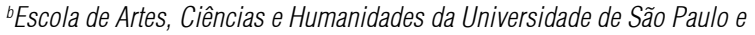 \\ Centro de Estudos da Metrópole. São Paulo, SP, Brasil. \\ E-mail: renatabichir@gmail.com \\ Orcid: 0000-0003-3111-2390
}

http://dx.doi.org/10.1590/0102-211246/113

\section{Introdução}

Com frequência nos deparamos com o surgimento de políticas com contornos similares em locais muito distintos, resultado de processos de difusão e/ou de transferência de

1 Agradecemos ao CNPq pela bolsa de doutorado concedida à Maria Clara Oliveira. Agradecemos também à FAPESP pelo financiamento atribuído ao projeto "Governança multinível em políticas sociais nacionalmente estruturadas: o caso da assistência social", coordenado por Renata M. Bichir, no Centro de Estudos da Metrópole (CEM/CEPID/Fapesp). Gostaríamos ainda de agradecer os pareceres de dois avaliadores anônimos da Lua Nova, cujos contributos foram essenciais para aprimorar o artigo. Igualmente valiosos foram os comentários de Osmany Porto de Oliveira e de Michelle Sá Silva a uma versão prévia deste artigo, apresentada no II ICPDDC. 
modelos de políticas públicas. ${ }^{2}$ Orçamento participativo, transferências monetárias, políticas para a primeira infância, são alguns exemplos de iniciativas que têm sido replicadas em vários contextos. Mantendo-se algumas semelhanças nos princípios orientadores dessas políticas, é possível observar diferenças consideráveis nos desenhos e nos modos de implementação. Apesar de uma parte da literatura recente (por exemplo, Peck e Theodore, 2015) mencionar que o processo de transposição de um modelo pode causar alterações na política pública, esse aspecto ainda é, com muita frequência, desconsiderado nos estudos sobre difusão de políticas públicas, que examinam apenas processos de mudança de agenda e de formulação, sem se atentar para as especificidades do desenho e para os processos de implementação.

Em oposição, encontramos estudos que se concentram na entrada na agenda e nos processos de formulação de políticas públicas, ignorando possíveis influências externas. Entretanto, face a um crescente grau de interdependência entre unidades e na presença de adesões múltiplas a políticas públicas semelhantes, torna-se imperativo pensar mais além. Entre o vasto grupo de estudos de análise de política pública, destacamos o grupo de abordagens cognitivas, que atribuem às ideias um papel central. Ideias são "crenças causais e normativas detidas por atores políticos e sociais" (Béland e Mahon, 2016, p. 43), que podem ser subdivididas, segundo Mehta (2011), em: (1) Zeitgeist ou ideias abrangentes; (2) ideias a respeito de problemas, que correspondem à identificação de uma questão; e (3) ideias a respeito de soluções, que aparecem para fazer face a um problema. As abordagens cognitivas incluem vários modelos, que mobilizam de modos distintos a importância das ideias nos processos de definição de agenda e de formulação de política pública - modelo de

2 Para mais informações sobre processos de difusão e de transferência de políticas públicas consultar Marsh e Sharman (2010) e Dolowitz e Marsh (2000), por exemplo. 
múltiplos fluxos de Kingdon (1984), modelo do equilíbrio pontuado, Advocacy Coalition Framework (ACF) de Sabatier e Jenkins-Smith (1993) e suas evoluções (Sabatier e Weible, 2007), mudanças de paradigmas de Hall (1993), abordagens de referenciais de Muller (2011).

Recorrendo isoladamente aos estudos da difusão e aos modelos de análise de políticas públicas, temos apenas uma visão parcial desses fenômenos, olhando ora a circulação de modelos, ora o nascimento e subsequente vida das políticas. Oliveira (2018) argumenta que a criação de uma lente de análise mais potente para estudar políticas globais passa pelo uso combinado das abordagens mencionadas. A autora sugere ainda que os conceitos de comunidade epistêmica conjunto de atores unidos em torno de uma ideia problema e instrument constituency ${ }^{3}$ - grupo reunido em torno de uma ideia solução -, permitem combinar as duas literaturas.

Este artigo analisa a entrada da ideia de transferência monetária na agenda e o surgimento de programas em dois contextos específicos, o Brasil e o Chile, demonstrando a utilidade dos conceitos comunidade epistêmica e instrument constituency para entender o que ocorre no plano local. O Brasil e o Chile foram selecionados por terem programas que servem de modelo para a América Latina e para outras regiões. Também constituem casos interessantes do ponto de vista da transformação de uma mesma ideia genérica em diferentes desenhos de políticas públicas. A partir do estudo desses dois casos e da análise do modo como ideias e fatores políticos e institucionais influenciam as estratégias ao longo do tempo, mostramos a existência de uma instrument constituency que promove a transferência monetária como

\footnotetext{
3 Uma possível tradução de instrument constituency é "grupo de defesa de instrumento de política pública", como usado por Howlett et al (2008). No entanto, optamos por manter o termo em inglês, uma vez que nos parece que esta tradução restringe a ação destes grupos à defesa de um instrumento, enquanto ela também inclui outros aspectos, como a sua discussão e aprimoramento.
} 
solução e atua no (re)desenho e implementação desse tipo de intervenção.

Por transferência de renda entende-se o seguinte tipo de intervenção: "noncontributory transfers of cash from formal institutions to targeted individuals or households" (Garcia e Moore, 2012, p. 3). Desde meados dos anos 1990, as transferências monetárias, que podem ter formatos distintos (ter ou não condicionalidades, por exemplo), têm-se firmado como um importante instrumento de combate à pobreza e à vulnerabilidade, estando presentes num número crescente de países, em particular na América Latina, África Subsaariana e Sudeste Asiático. Diversos estudos procuraram explicar a reprodução desses programas no Sul Global (Olivier de Sardan, 2018; Silva, Kauchakje e Delazari, 2017; Sugiyama, 2011; Leite e Peres, 2013; Leite, Peres e Belix, 2013; OsorioGonnet, 2015, 2018; Foli, Béland e Fenwick, 2017; Howlett, Ramesh e Saguin, 2018; Béland et al., 2018; Borges, 2018; 214 Osorio-Gonnet, Oliveira e Vergara, 2020; Osorio-Gonnet e Vergara, 2019), examinando os atores e os canais que contribuem para o processo de disseminação do modelo. Esses estudos destacam a existência de um conjunto variado de atores - como consultores do Banco Mundial, representantes de agências de cooperação para o desenvolvimento, acadêmicos e outros - que contribuem para a difusão e para a transferência dessas políticas, por meio da discussão de ideias, da produção e disseminação de informação a respeito dos programas existentes, da facilitação do contato entre diferentes partes e por meio da atribuição de financiamentos, entre outros.

A ampla disseminação desses programas é, por vezes, enquadrada como parte de vasto processo de financeirização da política social e de adoção de paradigmas neoliberais de proteção, a exemplo da argumentação presente em Lavinas (2017). Defendemos, entretanto, que o uso ampliado e por vezes impreciso de termos como "financeirização" e 
"neoliberalismo" tende a sobrevalorizar macrodinâmicas sem a devida análise da tradução local desses paradigmas e de outras contratendências, como alguns autores têm apontado (Ong, 2006; Lanzara e Bichir, 2019). Por outro lado, mesmo concordando com as inúmeras análises que apontam limites aos programas de transferência de renda como instrumentos de combate à pobreza e à desigualdade e a importância de sua articulação com outras políticas sociais universais (ver, por exemplo, Kerstenetzky, 2017), ressaltamos que não pretendemos, nesse texto, entrar na ampla e consolidada discussão sobre a avaliação dos impactos dessas políticas.

Criado em 2003, o programa brasileiro Bolsa Família tem vários objetivos: combater a fome e garantir a segurança alimentar e nutricional; o combate à pobreza e à privação; o acesso à rede de serviços públicos, especialmente de saúde, educação e assistência social; e contribuir para a emancipação das famílias e para o desenvolvimento local (Cotta, 2009; Monteiro, 2011). Enquanto as famílias em extrema pobreza recebem o Benefício Básico (transferência pura), às famílias pobres com crianças e/ou adolescentes é atribuída uma transferência com condicionalidades nas áreas de educação e saúde. O incumprimento das condicionalidades faz com que a família seja advertida e pode levar à suspensão do benefício, caso a situação se mantenha.

O Chile Solidario, criado em 2002, destinava-se a famílias extremamente pobres e tinha por objetivo ligar os beneficiários à rede de apoio do Estado. A participação no Chile Solidario incluía uma etapa intensiva (dois anos) a que se seguia a fase de acompanhamento (três anos). Primeiro, as famílias integravam o Puente, um programa de acompanhamento psicossocial, ${ }^{4}$ no qual uma estratégia de ação para a superação da pobreza era definida conjuntamente, entre

\footnotetext{
${ }^{4}$ Um assistente social acompanhava a família, procurando identificar debilidades e formas de as mitigar, em sete dimensões de bem-estar: habitação, saúde, renda, educação, registo, emprego e dinâmicas intrafamiliares (Barrientos, 2010).
} 
a família e o Estado (na figura do assistente social), tendo em consideração necessidades e preferências específicas. Durante esse período, as famílias recebiam uma transferência, cujo valor decrescia com o tempo e que visava apoiar a realização das metas, além de garantir também o acesso preferencial a alguns subsídios. Na fase de acompanhamento, as famílias recebiam o Bono de Egreso, uma transferência incondicional. Apesar das avaliações positivas, o Chile Solidario foi descontinuado e substituído pelo Ingreso Ético Familiar. ${ }^{5}$

Este artigo baseou-se numa revisão da literatura, complementada com análise de documentos oficiais e entrevistas. As entrevistas foram realizadas com indivíduos centrais para compreender a formulação dos programas, como acadêmicos, burocratas de médio e alto escalão, representantes de organizações da sociedade civil e de organizações internacionais e agências doadoras. Foram inicialmente estabelecidos contatos com indivíduos com participação direta nos progra216 mas - por exemplo, nomes mencionados em documentos oficiais e/ou em produções acadêmicas. Em seguida, utilizou-se uma estratégia de bola de neve, técnica que consiste em pedir aos entrevistados que referenciem outros indivíduos que consideram relevantes, de modo a identificar outras pessoas a entrevistar. Considerou-se que o espaço para realização de novas entrevistas se esgotou quando começaram a se repetir nomes e informações. Considerou-se também que a triangulação dessas informações e referências permitiu identificar os principais membros das comunidades epistêmicas e instrument constituencies em análise.

As entrevistas seguiram um roteiro semiestruturado adaptado a cada entrevistado, foram realizadas em

\footnotetext{
${ }^{5}$ Em 2012, o Ingreso Ético Familiar substituiu o Chile Solidario, mantendo parte das suas características, nomeadamente a transferência monetária e a existência de um plano de metas específico acordado entre a família e o Estado. Entre as novidades encontram-se uma abordagem voltada para a inserção no mercado laboral formal e a instituição de diversos "prêmios". Para mais informações ver, por exemplo, Oliveira (2018) e Oliveira e Osorio-Gonnet (no prelo).
} 
português ou em espanhol, e aconteceram presencialmente ou por telefone ou Skype nos casos em que não foi possível viabilizar um encontro. As informações sobre o Brasil foram recolhidas no período que se estende de outubro de 2015 a outubro de 2017, enquanto as entrevistas realizadas no Chile aconteceram nos meses de outubro e novembro de 2016 e em abril de 2017. Foram realizadas 34 entrevistas com enfâse nas experiências brasileira e chilena. Somaram-se ainda 7 entrevistas com consultores e representantes de organizações internacionais, realizadas na Inglaterra, entre janeiro e março de 2017, cujo foco foi a recolha de informações sobre programas de transferência monetária, de um modo geral. Com o intuito de minimizar possíveis constrangimentos resultantes da gravação das entrevistas, optou-se por tomar notas manualmente. As identidades dos entrevistados não serão reveladas ao longo do texto.

\section{Distinguindo comunidades epistêmicas de instrument constituencies}

Comunidade epistêmica é um conceito proposto por Haas (1992, p. 3), que a define como "a network of professional with recognized expertise and competence in a particular domain and an authoritative claim to policy-relevant knowledge within that domain or issue area". Um grupo constitui uma comunidade epistêmica quando: (1) existem normas e crenças que servem para aglutinar os membros; (2) há reconhecimento dos mesmos mecanismos causais; (3) são utilizadas noções de validação similares; e (4) a mesma visão sobre práticas e políticas públicas (Haas, 1992). As comunidades epistêmicas possibilitam a circulação de ideias e procuram influenciar a condução das políticas públicas, por meio do fornecimento de informação, havendo uma relação direta entre a sua capacidade de intervenção e a incerteza relacionada ao tema (Haas, 1992).

A proposta de Haas (1992) foi revista por diferentes autores que procuraram aprimorar o conceito em diversos 
aspetos. Para Cravinho (2006) a definição de Haas “[...] reduz a comunidade epistêmica às ideias partilhadas por um grupo de cientistas ou técnicos...”. O autor sugere que comunidades epistêmicas deveriam ser entendidas como "comunidades unidas por crenças relativas ao comportamento de indivíduos e grupos em sociedade" (Cravinho, 2006, p. 252). Cross (2013) também nota que os trabalhos que aplicam o conceito tendem a entendê-lo como um grupo composto exclusivamente por acadêmicos ou especialistas. No nosso entender, as comunidades epistêmicas são compostas por especialistas, que podem estar vinculados à esfera acadêmica ou ter outro tipo de atuação (por exemplo, representantes de organizações internacionais, burocracias especializadas, entre outros), com conhecimento acumulado sobre um determinado tema, fruto da reflexão e do trabalho desenvolvido e cuja expertise é reconhecida pelos pares. Seguindo essa linha, num exercício de mapeamento 218 da comunidade epistêmica regional ligada às transferências monetárias na América Latina, Osorio-Gonnet (2018) demonstra que esta é composta não só por acadêmicos, mas também por burocratas de alto-escalão e por consultores de organismos internacionais e de instituições financeiras.

A formulação de Haas (1992) baseia-se no entendimento de que as comunidades epistêmicas têm absoluto controle sobre o conhecimento e a informação sobre um determinado tema e isto confere-lhes autoridade para conduzirem os caminhos das políticas públicas que a ele se referem (Dunlop, 2009). Dunlop (2009) afirma que a capacidade dessas comunidades de manterem um controle total do conhecimento sobre o tema pode variar em função do espaço e do tempo. A autora avança a discussão ao construir uma tipologia para classificar a influência das comunidades epistêmicas com base nos tipos de aprendizagem. A possibilidade de influência e, por conseguinte, de difusão e transferência de modelos, advém de assumirem uma posição de comando relativamente 
ao conhecimento, que lhes permite apresentar-se como capazes de prover orientações para os decisores.

Na proposta inicial, as comunidades epistêmicas desempenham um papel de particular importância em temas com um grau de incerteza alto (Haas, 1992). Entretanto, elas podem também ser mobilizadas para apoiar e legitimar as decisões que um governo pretende tomar (Dunlop, 2009). Mais ainda, diante de políticas polêmicas, caso a opinião da comunidade epistêmica contrarie as escolhas dos decisores, estes podem procurar conhecimentos alternativos que melhor sustentem as suas opões, diminuindo drasticamente a margem de atuação da comunidade (Dunlop, 2017).

Além disso, Löblová (2017) afirma que a presença de uma comunidade epistêmica não é suficiente para explicar mudanças na condução das políticas públicas. A autora mostra que a sua influência pode ser interrompida a qualquer momento por motivos diversos (mudança de governo e surgimento de outras prioridades, por exemplo), tornando-se necessário atentar para outros fatores.

Um novo conceito surge nos últimos anos, o de instrument constituency. Trata-se de grupos, compostos por atores variados, que se constituem ao redor de um determinado instrumento e por causa dele (Simons e Voß, 2018), e cuja atuação é pautada pelo intuito de aprimorar, disseminar e viabilizar a adoção de um instrumento de política pública (Voß e Simons, 2014). De acordo com Béland, Howlett e Mukherjee (2017) comunidades epistêmicas são diferentes de instrument constituencies, uma vez que as primeiras agem no sentido de dar visibilidade a um problema e de o colocar na agenda política, enquanto a preocupação das segundas reside em ofertar aos decisores uma determinada ferramenta, provendo informação para que eles possam colocá-la em prática.

Partindo da distinção proposta por Mehta (2011) de ideias-problema e ideias-solução, podemos pensar 
as comunidades epistêmicas como um grupo promotor de ideias-problema, enquanto as instrument constituencies se unem em torno de ideias-solução (Oliveira, 2018). Essa proposta está alinhada com a sugestão de Béland e Howlett (2016) e de Béland et al. (2017) de utilizar as comunidades epistêmicas e as instrument constituencies para aprimorar o modelo de múltiplos fluxos de Kingdon (1984), no qual é necessária a convergência do fluxo de problemas com o das soluções e o da política, para que uma janela de oportunidades se possa abrir e, consequentemente, exista a possibilidade de mudança. Segundo os autores, comunidades epistêmicas agem com o intuito de colocar um problema na agenda, pelo que podem ser consideradas atores ligados ao fluxo de problemas, enquanto instrument constituencies se mobilizam em torno de ideias-solução, participando do fluxo de soluções, ou seja, analiticamente é possível separar os dois grupos em função do tipo de ideia que 220 os une e move. No entanto, esta distinção não significa que não haja eventual sobreposição empírica, ou seja, pode não haver uma diferença clara entre comunidade epistêmica e instrument constituency em casos em que o problema e a solução são promovidos conjuntamente. Assim, a operacionalização desses dois conceitos e a sua articulação no plano empírico varia de caso para caso e requer investigação adicional - o presente estudo comparativo procura iluminar essas questões.

A presença de um número extenso de ideias a respeito de problemas e de soluções traduz-se na existência de várias comunidades epistêmicas e instrument constituencies. Como cada grupo procura obter o monopólio (Haas e Haas, 1995) pode haver competição (Howlett Ramesh e Saguin, 2018). A competição pode ainda vir de dentro da própria comunidade epistêmica ou instrument constituency, dado que o acordo se dá relativamente às macro ideias, podendo haver divergência quanto a ideias secundárias - também 
Sabatier e Weible (2007) chamam a atenção para a possibilidade de divergências quanto a ideias secundárias dentro das coalizões de interesse.

Os atores que intervêm no processo de disseminação de ideias e práticas podem ser bastante distintos (Stone, 2004; Karch, 2007). Organizações internacionais e instituições financeiras internacionais desempenham um papel relevante, pois produzem e difundem conhecimento (Stone, 2012), além de terem capacidade de influência por meio da prestação de apoio técnico e/ou financeiro aos estados nacionais. Olivier de Sardan (2018) destaca a sua importância em processos de identificação de experiências que, depois de passarem por um processo de descontextualização e de "reconstrução" para ressaltar características chave e facilmente replicáveis, são apresentadas como histórias de sucesso e disseminadas. Acadêmicos e organizações da sociedade civil (OSC), entre outros, também atuam no refinamento de ideias e na sua promoção e, em certos casos, na tradução de ideias vindas de fora para o contexto local e/ ou na divulgação de ideias locais em outros contextos. Esses atores podem ser reagrupados em comunidade epistêmica e/ou instrument constituency, em função das ideias defendidas, ou seja, importa entender se são promovidas ideias-problema ou ideias-solução para determinar a que grupo pertencem os atores.

Campbell (2002) afirma que as comunidades epistêmicas são responsáveis pela criação de novas ideias e pela sua difusão no plano internacional. A existência de comunidades epistêmicas (Osorio-Gonnet, 2015, 2018) ou de redes de profissionais (Sugiyama, 2011) tem sido apontada como explicação para a introdução de políticas públicas já em vigor noutros contextos. Já sobre as instrument constituencies, a utilidade do conceito para entender processos de difusão e de transferência, além de processos locais, também é avançada por Béland et al. (2018). Foli et al. (2018) 
recorrem às instrument constituencies para explicar processos de transferência de política pública e consideram que têm um papel central dado que se engajam na produção e divulgação de informações e de conhecimento, fomentam debates em torno de um tema e facilitam a comunicação entre diferentes atores.

Utilizar os conceitos de comunidade epistêmica e de instrument constituency permite-nos costurar a circulação de ideias internacionalmente com a sua entrada nas agendas nacionais (Oliveira, 2018). No entanto, é preciso ter em consideração que as ideias são moldadas por uma série de outros fatores, o que torna necessário um olhar para outros elementos, como as políticas prévias, e as disputas políticas.

\section{Usos prévios dos conceitos de comunidades epistêmicas e instrument constituencies no estudo das transferências monetárias}

222 De que modo a literatura tem mobilizado os conceitos de comunidades epistêmicas e instrument constituencies para abordar o tema das transferências monetárias? OsorioGonnet $(2015,2018)$ aponta a existência de uma comunidade epistêmica regional responsável pelo surgimento de transferências monetárias condicionadas em um curto espaço de tempo em quase todos os países da América Latina e do Caribe. A autora demonstra a existência de um grupo de especialistas reunidos em torno da ideia de transferência condicionada como opção para combater a pobreza, identificando os membros do grupo (representantes de organizações regionais e internacionais, especialistas, entre outros), os diferentes momentos e espaços de interação e os recursos mobilizados. A partir do estudo dos programas chileno e equatoriano, a autora demonstra que há uma relação entre o nível de capacidade estatal e o tipo de mecanismo de difusão presente - quando é elevada, o mecanismo é a aprendizagem; se é baixa, observa-se a presença de mecanismos de 
coerção e de emulação (Osorio-Gonnet, 2018). Partilhamos da visão de Osorio-Gonnet (2018) sobre a existência de um grupo de atores que se mobilizam ao redor da transferência monetária e com presença ativa na América Latina, mas sugerimos denominá-lo de instrument constituency ao invés de comunidade epistêmica, uma vez que ele se reúne em torno de uma ideia-solução.

Foli, Béland e Fenwick (2018) usam o conceito de instrument constituency para explicar a transferência do Bolsa Família para Gana, que teria facilitado o processo de transferência ao atuar na "produção de conhecimento sobre os instrumentos e a sua performance, ao promover um determinado discurso em encontros internacionais, ao facilitar a aprendizagem e ao ofertar recursos aos países que manifestassem interessem em pilotar programas representativos de um certo tipo de instrumento" (Foli, Béland e Fenwick, 2018, p. 11, tradução nossa). Howlett et al. (2018) atribuem a adoção de uma transferência condicionada nas Filipinas à ação de uma instrument constituency, que teria promovido o instrumento por meio de narrativas e da produção de informação a seu respeito. Ao comparar os casos filipino e ganês sobre a adesão às transferências monetárias, Béland et al. (2018) assinalam a importância de instrument constituencies, compostas por atores variados (ex. representantes de organismos internacionais, consultores e outros), no refinar do modelo e na sua disseminação e tradução em diferentes contextos.

Os estudos sobre o tema centram-se na ação das comunidades epistêmicas e das instrument constituencies no plano internacional e/ou na passagem do plano internacional para o plano nacional. Reconhecendo a importância da atuação desses grupos nas esferas regional e internacional e também a existência de ligações entre os planos externos e internos, neste artigo, abordamos o papel desempenhado por estes grupos na esfera doméstica, analisando concretamente os casos do Brasil e do Chile. 


\section{A adoção das transferências monetárias condicionadas no Brasil}

Outros estudos já haviam analisado os grupos que participaram da formulação do Programa Bolsa Família. Tomazini (2010), que aplica o ACF de Sabatier e JenkinsSmith (1993), identificou três coalizões de interesse - renda básica universal, segurança nutricional e alimentar e desenvolvimento de capital humano -, sendo a criação do Bolsa Família um indicador da força da última. Por sua vez, Cotta (2009) dedicou-se à identificação dos grupos que fazem parte da construção do programa e à discussão das visões de pobreza detidas por cada um deles. Já Silva (2010) entende o Bolsa Família como o resultado de uma visão dominante sobre como se deve proceder no combate à pobreza. Se estes trabalhos são avanços no sentido de compreender como se dá o processo de formulação interno e que grupos intervêm, eles apresentam algumas limitações, dado que, à exceção 224 do estudo de Silva (2010), os modelos usados desconsideram que a adoção do programa faz parte de um processo de difusão.

No Brasil, a associação de transferências a condicionalidades na área da educação ganhou força no começo dos anos 1990, debate liderado por Cristovam Buarque e pelo seu grupo de estudos na Universidade de Brasília. Nas palavras de Buarque (2012, p. 14): "use-se a necessidade de renda dos pobres para induzi-los a colocar seus filhos na escola".

Experiências emblemáticas nessa área surgiram, primeiro, nos níveis subnacionais: o Bolsa Escola no Distrito Federal e o Programa de Garantia de Renda Mínima Familiar em Campinas. Anunciado durante a campanha eleitoral por Buarque, candidato a governador do Distrito Federal, e implementado em janeiro de 1995, o programa brasiliense consistia-se numa transferência monetária, cuja contrapartida era a frequência escolar de crianças de 7 a 14 anos e à qual se juntaram outros componentes, como 
uma poupança para os estudantes, um programa de alfabetização de adultos e a entrega de brinquedos. ${ }^{6}$ Avaliações, em particular da UNICEF e do Banco Mundial, destacaram os resultados positivos e aumentaram a visibilidade do programa (Buarque, 2012). Em Campinas, a discussão decorreu da preocupação com questões de segurança alimentar (Leite e Peres, 2013, entrevista). A experiência campineira condicionava o benefício à realização de atividades nas áreas de educação e saúde (Coelho, 2012).

Esses programas foram rapidamente emulados por outros municípios e governos estaduais. Há várias explicações para a rápida disseminação deste instrumento: competição política (Coelho, 2008), proximidade ideológica (adesão por partidos de esquerda) e existência de uma rede de profissionais que valorizava a transferência condicionada (Sugiyama, 2008a, 2008b). Para um entrevistado, a adoção acontecia “[...] muito mais por imitação do que por avaliação de resultados, por prestígio pessoal" (entrevista). A Lei Federal nº 9.533 de 1997 constituiu um impulso aos programas locais, ao conceder apoio financeiro aos municípios para implementação de transferências condicionadas: não dispondo o município de recursos suficientes, a União podia cobrir até $50 \%$ dos custos (Veras Soares, 2011, Bichir, 2011).

Na esfera federal, o primeiro programa de transferência condicionada data de 1996, o Programa de Erradicação do Trabalho Infantil (PETI), que previa um benefício para famílias com crianças em situação de trabalho infantil, mediante a sua retirada do mercado de trabalho e permanência na escola (Veras Soares, 2011). Mais tarde, em 2001, o Programa Bolsa Escola (PBE) foi adotado pela União, sendo uma reprodução parcial do desenho da iniciativa do Distrito Federal. A transferência, via cartão, atingia até 3 crianças por família e exigia frequência escolar não

${ }^{6}$ Para informações adicionais sobre o programa de Brasília consultar Buarque (2012). 
inferior a $85 \%$ (Coelho, 2012). Os municípios que aderiam ao programa deviam identificar as famílias beneficiárias e desenvolver ações socio-educacionais. Ainda nesse ano, foi criado o Cadastro Único (Cadúnico), até hoje usado para recolher informação sobre as famílias brasileiras em situação de pobreza. As transferências monetárias multiplicaram-se durante o governo Fernando Henrique Cardoso - Bolsa Gás, Bolsa Alimentação - traduzindo um apoio a este tipo de intervenção. Entretanto, o fato de os programas terem diferentes públicos, financiamentos distintos e serem da responsabilidade de vários ministérios, resulta numa rede de proteção esgarçada, com problemas de focalização (ao mesmo tempo que há sobreposição de públicos, existem grupos desprotegidos) e com um sério grau de desarticulação.

A chegada de Lula ao poder, em 2003, trouxe a estra-

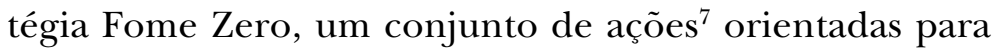
o combate à fome e à desnutrição. Entre o rol de iniciativas encontrávamos o Cartão Alimentação, uma transferência destinada à compra de alimentos. O combate à fome assumiu um lugar central nesse momento, como indicado pela criação do Ministério Extraordinário de Segurança Alimentar (MESA).

Ainda no período de transição para o governo Lula, formou-se um grupo de especialistas para identificar temas de ação prioritária e foi abordada a existência de múltiplos programas de transferência condicionada em paralelo (Monteiro, 2011). O relatório apresentado apontava a vantagem de unificar os programas e a urgência no combate à fome e à insegurança alimentar; sugeria criação de um cartão para efetuar pagamentos e mencionava a necessidade alterações ao CadÚnico (Monteiro, 2011; Cotta, 2009, entrevista). A Câmara de Política Social (CPS), criada no

\footnotetext{
7 A estratégia Fome Zero incluía ações variadas, tais como o acesso ao microcrédito, bancos alimentares, iniciativas de apoio à agricultura familiar, entre outras.
} 
começo do mandato com o objetivo de coordenar as ações da área social, apresentou um diagnóstico semelhante ao da equipe de transição (Monteiro, 2011). O Ministério da Fazenda também demonstrou preocupação com a focalização (Monteiro, 2011).

Um grupo técnico foi constituído especificamente para cuidar das políticas sociais e apresentou então as seguintes soluções para os problemas levantados no relatório: (1) o CadÚnico devia tornar-se um instrumento central para identificação dos beneficiários; (2) os programas deviam ser fundidos em uma só medida; e (3) os pagamentos deviam ser feitos por intermédio de um cartão (Monteiro, 2011). Havia um consenso sobre ao formato do programa - a opção era por uma transferência condicionada (Cotta, 2009) - o que revela a presença de membros da instrument constituency a favor dessa opção dentro do grupo técnico. Com efeito, nesse momento a ideia de transferência condicionada já era amplamente discutida em vários espaços, além de existir um número muito grande de experiências em prática nos vários níveis de governo e, consequentemente, um acúmulo de conhecimento a respeito do instrumento (conhecimento que se soma ao proveniente de outras experiências latino-americanas). Note-se que alguns dos indivíduos que compõem o grupo técnico tiveram, inclusive, contato com experiências desenvolvidas localmente, como ressaltado por Leite e Peres (2013) e em entrevistas.

Esse momento foi marcado pela apresentação de propostas distintas por parte do grupo que compõe a CPS e pelo MESA. Após diversas reuniões com a presença do grupo técnico e de representantes dos ministérios, surgiu uma nova proposta. Uma instrument constituencyé tida como um grupo homogêneo e com uma opinião coesa sobre a importância de um instrumento, no caso a transferência condicionada. Contudo, isso não quer dizer que exista dentro do grupo um consenso absoluto e, portanto, uma ausência de discussão 
em relação a qualquer ponto. A associação faz-se em torno de ideias macro, de um núcleo duro - nesse caso, em torno da ideia de associar à transferência condicionalidades com ênfase no desenvolvimento de capital humano -, mas pode existir desacordo quanto a ideias mais secundárias, de operacionalização do programa - características do públicoalvo ou valores, por exemplo. A apresentação de diferentes propostas de desenho do programa ilustra bem os embates que podem ocorrer entre membros de uma instrument constituency em relação a ideias secundárias. A nova proposta continha as seguintes linhas: (1) unificação dos programas; (2) os objetivos definidos eram o combate à fome, a garantia de segurança alimentar e nutricional e a diminuição da pobreza; (3) a transferência estava associada a exigências de educação e saúde, de modo a incentivar o desenvolvimento de capacidades; (4) a verificação do cumprimento das condicionalidades era feita pelos órgãos responsáveis pela 228 provisão desses serviços; (5) em caso de não cumprimento, a família seria advertida e, se a situação se mantivesse, podia ser excluída do programa; (6) a gestão do programa era feita pelos três níveis de governo; (7) o programa continha dois benefícios distintos: (a) uma prestação incondicional com montante fixo para famílias extremamente pobres, e (b) transferência para famílias em situação de pobreza e com crianças/adolescentes, cujo valor variava em função da composição do agregado familiar; (8) os pagamentos eram mensais e via cartão; (9) a transferência era feita preferencialmente para mulheres; (10) o programa era nacional e a sua implementação dava-se em função do orçamento disponível; (11) os beneficiários eram identificados pelos municípios, seguindo critérios definidos pela União (Monteiro, 2011). Na sequência dessa apresentação, o MESA propôs novamente um projeto alternativo (Monteiro, 2011).

Um novo projeto começou a ser elaborado, com o apoio de Lula e com a premissa de que se deveria procurar 
construir uma intervenção única e capaz de abrigar os diferentes setores envolvidos (Monteiro, 2011). O grupo de trabalho foi composto por representantes de múltiplos órgãos e subdividido em quatro subgrupos temáticos - desenho e implementação do novo programa, CadÚnico, orçamento e legislação (Monteiro, 2011).

A proposta final nasceu em agosto de 2003, sendo o programa anunciado nas semanas seguintes, com a implementação a iniciar-se em outubro e manutenção dos programas anteriores até à migração de todos os beneficiários. O Programa Bolsa Família sintetizava os vários objetivos que estavam a ser disputados até então - diminuição da pobreza, desenvolvimento de capacidades, combate à fome - por meio de uma transferência condicionada. Os programas anteriores desapareceram, mas o núcleo duro das ideias em que se baseavam fora mantido e integrado ao Bolsa Família, alterando-se as ideias secundárias dos programas e os motivos que levavam à mobilização desse instrumento, ou seja, o objetivo passou a ser a garantia de um patamar mínimo no plano imediato e a quebra da transmissão intergeracional de pobreza, em virtude do desenvolvimento de capacidades resultantes de maiores investimentos em capital humano, o que justificou novas condicionalidades na área da saúde.

A importância do apoio direto do presidente no sentido de viabilizar a criação de uma solução comum e satisfatória para todos os envolvidos chama a atenção para um ponto que já havia sido notado por Löblová (2017) sobre as comunidades epistêmicas, mas que pode ser transposto para as instrument constituencies: por si só a ação desses grupos não é condição suficiente para transformar ideias (problemas ou soluções) em ações concretas, sendo necessário atentar-se também para outros fatores, como questões políticas. Nesse caso, comunidade epistêmica e instrument constituency sobrepõem-se - de um lado o grupo preocupado com a questão da pobreza propõe uma transferência condicionada e 
de outro o grupo preocupado com a segurança alimentar sugere a aplicação de instrumentos alternativos, em meio aos quais se encontra o Cartão Alimentação, também ele uma transferência, porém incondicional. $\mathrm{O}$ apoio presidencial e os esforços do grupo de trabalho permitiram a adoção do instrumento defendido por uma das instrument constituencies, escolhido com o intuito de responder às preocupações manifestadas pelas duas comunidades epistêmicas, embora em menor escala à que promovia o tema da segurança alimentar. Ressalta-se ainda a existência de uma instrument constituency que propõe serviços socioassistenciais e que, mais tarde, obteve voz dentro do Ministério do Desenvolvimento Social (MDS).

Ainda que não tenha havido participação direta de atores externos na formulação do programa, como aconteceu noutros contextos, o processo não se deu de modo alheio a outras experiências. Além do aprendizado com as experiências 230 prévias em território brasileiro, existia também um conhecimento difuso sobre programas latino-americanos e há registro de missões que permitiram a troca de informações com atores envolvidos noutros programas. Com efeito, tanto Peck e Theodore (2015) como Silva (2010) afirmam que Santiago Levy, idealizador da transferência condicionada mexicana, e James Wolfensohn, então presidente do Banco Mundial, teriam encontrado Lula antes do lançamento do Bolsa Família. Anteriormente, tiveram também lugar em diversas missões de divulgação do Bolsa Escola e de contato com iniciativas similares (entrevistas). Além disso, antes do início das discussões que desembocaram na criação da medida, o Banco Mundial havia produzido recomendações a respeito da proteção social no Brasil, em linha com o que foi debatido pela equipe de transição (Silva, 2010, entrevista). A organização não participou das discussões, mas reconheceu no próprio dia do anúncio de criação do programa a importância de colocar em marcha tal iniciativa (entrevista). Nas palavras de 
um entrevistado "O Banco Mundial constrói, mas não intervém [...] Ele é um player muito importante. Não dá para viver sem ele. Ele tem o papel de trazer ideias". Mais tarde, o apoio traduziu-se também na forma de um empréstimo para a operacionalização do programa, que tinha um formato inovador, dado que colocava o Banco como provedor de apoio (entrevista). Esses exemplos mostram que existiam fluxos entre o braço da instrument constituency atuante no Brasil e um corpo mais amplo que atua na esfera internacional. Esses fluxos foram bidirecionais, já que não só o Brasil se beneficiou de conhecimentos sobre transferências monetárias a partir de debates e de experiências noutros contextos, como o Bolsa Família tornou-se um modelo, influenciando discussões em outras arenas.

A reestruturação que levou à criação do Bolsa Família encontrou algumas resistências, que não dizem respeito ao instrumento per se (entrevistas). Buarque (2012) argumenta que o programa retirou a centralidade da questão educativa para a colocar na transferência monetária. Com efeito, o setor de educação reconheceu a importância do programa, mas não o considerou uma medida da área educacional (Silva, 2010). Alguns dos ministérios anteriormente responsáveis por programas que desaparecem com a chegada do Bolsa Família também levantam objeções ao programa, pois encaram a fusão como uma diminuição de sua visibilidade (Monteiro, 2011).

\section{A criação das transferências monetárias condicionadas no Chile}

As transferências monetárias são há muito utilizadas no Chile como instrumento de combate à pobreza, existindo múltiplos programas que apoiam diferentes grupos carenciados, especialmente mulheres e crianças (Garay, 2010). O regime militar, instaurado em 1973, colocou em prática o modelo neoliberal, atribuindo um papel marginal 
ao Estado e reduzindo o gasto social (Raczinsky, 1994). O resultado foi um aumento do desemprego e dos níveis de pobreza. Nesse período, no plano da política social, destacou-se a reforma do sistema de pensões e a adoção de programas de workfare. É herança desse momento o Subsídio Único Familiar, implementado a partir de 1981, destinado a crianças (a idade máxima foi sendo expandida) e mulheres grávidas de famílias vulneráveis (Raczinsky, 1994) e que exigia como contrapartida consultas médicas (entrevista). Essa medida das demais prestações sociais pela condicionalidade pode ser considerada precursora da transferência condicionada no país (entrevista).

Com o regresso à democracia, multiplicaram-se as ações dirigidas a populações até então "invisíveis". Entre elas, destacou-se o nascimento do Fondo de Solidaridad $e$ Inversión Social (Fosis), que promove o acesso ao crédito e fornece apoio técnico para o desenvolvimento de projetos 232 e outros. Apesar da queda acentuada da pobreza durante os anos 1990, um estudo sobre as famílias chilenas em extrema pobreza conduzido em 1999, mencionava cerca de 225.000 famílias nesta situação e sem acesso às redes de apoio do Estado (Barrientos, 2010; Palma e Urzúa, 2005).

Uma das prioridades do governo de Lagos era o enfrentamento da extrema pobreza (Lagos, 2002) e o tema foi alvo de várias discussões, com participação de múltiplos atores, como membros de think tanks e de OSC e acadêmicos (Osorio-Gonnet, 2018). Dentro do Ministério do Planejamento (Mideplan) criou-se um grupo de trabalho, ao qual solicitou-se o desenho de uma estratégia voltada para as famílias em extrema pobreza. Como aconteceu no caso brasileiro, experiências prévias implementadas em esferas subnacionais constituíram uma fonte de inspiração. Foram elas: (a) o Programa Acciones Integradas para las Familias em Extrema Pobreza, aplicado em La Florida, com o qual havia trabalhado Veronica Silva, agora a cargo da coordenação 
do grupo; e (b) as experiências de combate à pobreza de Quillota (Osorio-Gonnet, 2018, 2020). Igualmente importantes foram os aprendizados retirados de iniciativas de apoio integral a famílias em situação de pobreza extrema desenvolvidas por duas OSC: a Fundación Rodelillo (FR) e o Hogar de Cristo (HdC) (entrevistas, Osorio-Gonnet, 2018, 2020). O resultado foi a criação do Programa Puente, uma estratégia de apoio psicossocial destinada a apoiar as famílias em situação de extrema pobreza por meio da definição conjunta de um plano específico, de acordo com as características da família. Tratava-se de um instrumento para garantir o acesso a serviços por parte das famílias que não tinham canais com a rede pública de serviços sociais (entrevista). Se esta iniciativa era bastante inovadora do ponto de vista dos serviços oferecidos pelo Estado, ela já estava presente em diversas OSC chilenas - no HdC, por exemplo, assistentes sociais visitam semanalmente as famílias atendidas, modelo por sua vez inspirado em práticas observadas nos Estados Unidos (entrevista). O apoio psicossocial também se beneficiou do fato de haver nesse momento, e nos anos que se seguiram, um número significativo de assistentes sociais no Mideplan, que sustentaram essa proposta (entrevista; Reininger et al, 2016). Mais tarde, o Puente tornou-se parte do Chile Solidario. A componente de apoio psicológico atraiu a atenção da comunidade internacional e foi replicada noutros países. ${ }^{8}$

De acordo com Franzoni e Voorend (2011), a OSC Asesorías para el Desarrollo teve um papel fundamental na criação de espaços de diálogo entre atores com posições muito diversas, permitindo costurar uma solução conjunta para responder ao problema da extrema pobreza. Entre as clivagens encontradas, podemos identificar um grupo que

\footnotetext{
8 Para mais informações sobre a reprodução dessa componente noutros contextos, consultar: Osorio-Gonnet, 2018; Osorio-Gonnet et al., 2020; Osorio-Gonnet e Vergara, 2019.
} 
insistia em investimentos em capital humano em oposição a outro que propunha a atribuição de vouchers (Palma e Urzúa, 2005). O desenho final resultou do encontro de duas frentes com propostas distintas: o Mideplan e da Dirección del Presupuesto del Ministerio de la Hacienda (Dipres). Por um lado, o Mideplan sugeriu a adoção do Puente, que já se encontrava em fase piloto (Osorio-Gonnet, 2018). Por outro, a Dipres, que trabalhava de modo articulado com o Banco Mundial, avançou a ideia de criar uma transferência monetária dirigida aos extremamente pobres (Osorio-Gonnet, 2018). Tal como no caso do Brasil, a capacidade de influência do Banco Mundial no Chile era restrita, em razão da existência de equipes técnicas fortes (Osorio-Gonnet, 2018). Assim, a instituição prestou apenas limitada ajuda técnica na formulação e, mais adiante, efetuou um empréstimo. De acordo com Osorio-Gonnet (2018), o anúncio de um programa de elevada complexidade, nomeadamente devido ao apoio psi234 cossocial, constituiu uma surpresa para os representantes do Banco Mundial.

A criação do Chile Solidario suscitou algumas manifestações de descontentamento. Não se tratava de uma rejeição do instrumento, mas de um desconforto relacionado à perda de competências por parte das OSC e, consequentemente, de recursos e de visibilidade. A proposta foi recebida por algumas das organizações com desconfiança (entrevista), mas a contestação foi feita de forma moderada, evitando criar tensões (entrevista). Essa questão reforça o ponto que já levantamos sobre a necessidade de considerar outros fatores, além da simples adesão ou não à ideia-solução.

Ainda que o Chile Solidario tenha sido apresentado pelo Presidente Lagos ao Congresso em maio de 2002 (Lagos, 2002), a discussão e aprovação da lei só aconteceu em 2004. A oposição aceitou o Chile Solidario após leves modificações do desenho (Garay, 2010), sendo a participação das comunas na implementação do programa um dos pontos 
de maior embate (BCN, 2002), o que sinaliza apenas uma discordância sobre a operacionalização do programa e não uma questão com o instrumento escolhido. O programa ficou a cargo do Mideplan, enquanto a gestão do Puente foi atribuída ao Fosis (Larrañaga e Contreras, 2010). A abordagem intersetorial implicava uma ação conjunta entre os dois órgãos, além de uma articulação com os Ministérios da Educação e da Saúde (Franzoni e Voorend, 2011), mas as ações eram frequentemente tomadas setorialmente (Palma e Urzúa, 2005; Larrañaga e Contreras, 2010). Um acordo estabelecido entre o Fosis e os municípios permitia a instalação de uma Unidad de Intervención Familiar (UIF), responsáveis pela coordenação do apoio psicossocial na esfera local (Larrrañaga e Contreras, 2010). O novo programa também foi recebido com entusiasmo pelas comunas, que frequentemente careciam de recursos para colocar em prática ações de combate à pobreza (entrevista).

À semelhança do que aconteceu com o Bolsa Família, a formulação do programa chileno foi conduzida de um modo relativamente isolado, contando com pouca participação de atores externos. Uma avaliação do programa piloto, na qual estavam inseridas algumas recomendações foi elaborado pela Comissão Econômica para a América Latina e o Caribe (Cepal) (Franzoni e Voorend, 2011). Já o Banco Mundial, como visto acima, assumiu uma posição de assessor e conduziu algumas avaliações (Larrañaga e Contreras, 2010), o que não significa, contudo, que o programa se desenvolveu sem qualquer influência externa, até porque já havia um acúmulo considerável de conhecimento, resultado de um processo difuso de aprendizagem, a respeito de programas colocados em prática noutros países latino-americanos (entrevistas).

Resumindo, a criação do programa resultou da interação de múltiplos atores: Mideplan, Ministério da Fazenda, representantes de OSC, acadêmicos, membros dos governos 
locais e alguns atores externos, como consultores do Banco Mundial (Franzoni e Voorend, 2011, entrevistas). O processo de formulação contou com a participação de um corpo técnico forte, dotado de vasto conhecimento sobre o tema (Osorio-Gonnet, 2015, 2018, 2020; entrevistas), o que explicaria o fato de o Chile conseguir desenhar o programa de modo relativamente isolado de influências externas e de forma extremamente inovadora, como o demonstra a introdução do componente de apoio psicossocial (OsorioGonnet, 2018).

A solução proposta no Chile para o problema da pobreza extrema é distinta das soluções de transferência condicionada até então encontradas no continente latino-americano por incluir um componente de apoio psicossocial e uma série de outras iniciativas, sendo mais central o trabalho do assistente social com as famílias do que o apoio monetário. Osorio-Gonnet (2018) identifica a presença de uma comuni236 dade epistêmica com atuação no Chile e atribui-lhe importância na formulação do Chile Solidario. Concordamos com a proposta da autora, mas acreditamos que o termo instrument constituency é mais adequado do que o de comunidade epistêmica por se tratar de uma ideia-solução. Este caso é interessante para mostrar que apesar de haver um consenso dentro da instrument constituency sobre a importância de um instrumento, há também espaço para que possa haver divergência e mudança nas ideias secundárias. Com efeito, no caso do programa chileno, podemos observar que existe um apoio à transferência monetária, mas que um segundo instrumento, o apoio psicossocial, defendido em particular por indivíduos provenientes da área de serviço social, é igualmente valorizado, dando origem a um desenho de política distinto daquele que vinha sendo implementado até então. A relação entre as discussões no plano doméstico e internacional faz-se por meio da ligação do braço local da instrument constituency a um grupo mais amplo, que atua na promoção 
dessas iniciativas. A formulação do Chile Solidario não conta com a participação direta de representantes dessa instrument constituency transnacional (por exemplo, representantes de organizações internacionais ou consultores internacionais), mas os policy-makers detinham conhecimento sobre experiências já em implementação (por exemplo, o México e experiências subnacionais brasileiras). Mais tarde, Banco Mundial, Banco Interamericano de Desenvolvimento e agências doadoras, que compõem a instrument constituency, tiveram um papel fundamental na disseminação da experiência chilena na América Latina. Além disso, policy-makers que participaram da elaboração e da implementação do Chile Solidario também constituíram veículos importantes de disseminação de informação sobre o programa chileno e na aprendizagem a partir de experiências em outros contextos.

\section{Considerações finais}

Para estudar o surgimento de políticas públicas em contextos distintos e em um curto período é necessário combinar estudos da difusão/transferência com abordagens cognitivas (Oliveira, 2018). Os conceitos de comunidade epistêmica e de instrument constituency permitem entender processos de difusão e podem também ser usados para iluminar os processos de formulação de políticas públicas (Oliveira, 2018; Béland et al., 2018). O objetivo deste artigo foi demonstrar que é vantajoso o uso desses dois conceitos para explicar a entrada na agenda e a formulação de políticas públicas globais em contextos nacionais, recorrendo para tal a um estudo empírico.

Neste trabalho analisamos a criação do Programa Bolsa Família e do Chile Solidario. Esses dois programas enquadram-se num processo de difusão internacional de um modelo de transferência monetária, tema amplamente trabalhado por outros autores e que não é objeto deste artigo. Seguindo a linha de Osorio-Gonnet (2018), argumentamos 
que a influência de atores externos é relevante, porém pautada pela "massa crítica" interna, ou seja, pela existência de comunidades epistêmicas e de instrument constituencies e por capacidades estatais previamente instaladas, as quais permitiram não só a formulação de programas, seguindo ondas de difusão do modelo, mas também a criação de instrumentos e soluções inovadores, posteriormente difundidos para outros contextos.

Os dois estudos de caso mostram a existência de uma instrument constituency com braços locais, reunida em torno da transferência monetária. Bolsa Família e Chile Solidario resultam da ação dessa instrument constituency e do seu sucesso na promoção do instrumento de transferência monetária, mas os desenhos distintos dos dois programas resultam de embates com outras comunidades epistêmicas/ instrument constituencies e de disputas dentro da própria instrument constituency, em torno de ideias mais secundárias e, 238 portanto, mais fáceis de ser alteradas (Hall, 1993) e adaptadas ao contexto local - para ilustrar, se no Brasil o Estado assume a responsabilidade pela situação das famílias, no Chile encontramos uma lógica de contratualização e de corresponsabilidade; se o Bolsa Família associa o benefício a condicionalidades de saúde e educação, no Chile Solidario o apoio psicossocial tem um lugar de destaque.

Enquanto categorias analíticas, é útil separar ideias por tipos e, consequentemente, distinguir entre comunidade epistêmica e instrument constituency. No plano empírico, a articulação entre esses dois grupos pode assumir diferentes formatos, que devem ser avaliados caso a caso. A análise dos dois países sustenta essa afirmação, na medida em que se observam diferenças entre os dois. Por um lado, no Brasil há uma sobreposição entre a comunidade epistêmica que defende o combate à pobreza e o investimento em capital humano com a instrument constituency constituída em torno do instrumento transferência monetária, ou seja, problema e 
solução são promovidos conjuntamente, havendo, contudo, disputas por espaço com outras comunidades epistêmicas e instrument constituencies (por exemplo, com grupos que atuam em torno da questão da segurança alimentar e promovem soluções alternativas). Além disso, esse caso ilustra a possibilidade de, dentro do mesmo grupo, existirem discordâncias no que concerne às ideias secundárias. Por outro lado, verifica-se no Chile a existência de uma comunidade epistêmica reunida em torno do tema da pobreza extrema e de várias instrument constituencies constituídas ao redor de diversos instrumentos, nomeadamente da transferência de renda e o apoio psicossocial, que são depois fundidos para criar um programa com componentes inovadoras.

As noções de comunidade epistêmica e de instrument constituency já foram usadas para entender processos de difusão ou de transferência de programas de transferência monetária (Osorio-Gonnet, 2018; Foli et al., 2018; Howlett et al, 2018; Béland et al., 2018). Este artigo procurou avançar a discussão, por meio da análise da elaboração do Bolsa Família e do Chile Solidario, ao demonstrar a adequação dos conceitos para entender os processos locais de adoção do modelo, contribuindo para fortalecer o argumento a favor da utilidade dos conceitos de comunidade epistêmica e de instrument constituency, para fazer a ponte entre as literaturas acima mencionadas.

\section{Maria Clara Oliveira}

Investigadora no Instituto de Direito Económico, Financeiro e Fiscal da Faculdade de Direito da Universidade de Lisboa e Pesquisadora Associada no Centro de Estudos da Metrópole. Doutora em Ciência Política pela Universidade de São Paulo.

\section{Renata Mirandola Bichir}

Professora na Escola de Artes, Ciências e Humanidades da Universidade de São Paulo e Coordenadora de Pesquisa e 
de Projeto no Centro de Estudos da Metrópole. Possui doutorado em Ciência Política (Ciência Política e Sociologia) pelo IESP-UERJ.

\section{Bibliografia}

BARRIENTOS, Armando. 2010. Protecting capability, eradicating extreme poverty: Chile Solidario and the future of social protection. Journal of Human Development and Capabilities. v. 11, n. 4, pp. 579-597. DOI: $10.1080 / 19452829.2010 .520926$

BÉLAND, Daniel; FOLI, Rosina; HOWLETT, Michael; RAMESH, M.; WOO, J.J. 2018. Instrument constituencies and transnational policy diffusion: the case of conditional cash transfers. Review of International Political Economy. v. 25, n. 4, pp. 463-482.

DOI: 10.1080/09692290.2018.1470548

BÉLAND, Daniel; HOWLETT, Michael; MUKHERJEE, Ishani. 2017. Instrument constituencies and public policy-making: an introduction. Policy and Society. v. 37, n. 1, pp. 1-13.

DOI: 10.1080/14494035.2017.1375249

BÉLAND, Daniel; MAHON, Rianne. 2016. Advanced introduction to social policy. London: Elgar Advanced Introductions, Edward Elgar Publishing.

BICHIR, Renata Mirandola. 2011. Mecanismos federais de coordenação de politicas sociais e capacidades institucionais locais: o caso do Programa Bolsa Família. Tese de Doutorado em Ciência Política e Sociologia. Rio de Janeiro: IUPERJ.

BORGES, Fabián. 2018. Neoliberalism with a human face? Ideology and the diffusion of Latin America's conditional cash transfers. Comparative Politics. v. 50, n. 2, pp. 147-167. Disponível em: https://bit.ly/3yUlx2u. Acesso em: 16 ago. 2021.

BUARQUE, C. 2012. Bolsa-Escola. História, Teoria e Utopia. Brasília, DF: Thesaurus Editora.

COELHO, Denilson Bandeira. 2012. Brazil - Basic Income - a new model of innovation diffusion. In: MURRAY, Matthew; PATEMAN, Carole. Basic Income Worldwide. Horizons of Reform. London: Palgrave Macmillan. pp. 59-80.

COÊLHO, Denilson Bandeira. 2008. A difusão do Programa Bolsa Escola: competição política e inovação no setor social. Trabalho apresentado no $32^{\circ}$ Encontro Anual da ANPOCS, Caxambu, 27 a 31 de outubro.

CAMPBELL, John L. 2002. Ideas, politics, and public policy. Annual Review of Sociology. v. 28, n. 1, pp. 21-38. DOI: 10.1146/annurev.soc.28.110601.141111 
COTTA, Tereza Cristina Silva. 2009. Visões de proteção social e transferência de renda condicionada no Brasil e no México. Tese de Doutorado em Estudos Comparados sobre as Américas. Brasília, DF: Universidade de Brasília. Disponível em: https://bit.ly/3g9nSzc. Acesso: 16 ago. 2021.

CRAVINHO, João Gomes. 2006 Visões do mundo. As relações internacionais e o mundo contemporâneo. 2. ed. Lisboa: ICS.

BCN - BIBLIOTECA DEL CONGRESO DE CHILE. Historia de la Ley n. 19.949. BCN. Santiago, 7 out. 2002. Disponível em: https://bit. ly/3sNi7si. Acesso em: 09 Jun. 2017.

CROSS, Mai'a K. Davis. 2013. Rethinking epistemic communities 20 years later. Review of International Studies. v. 39, n. 1, pp. 137-160. DOI: $10.1017 / \mathrm{S} 0260210512000034$

DOLOWITZ, David P.; MARSH, David. 2000. Learning from abroad: the role of policy transfer in contemporary policy-making. Governance: an International Journal of Policy, Administration and Institutions. v. 13, n. 1, pp. 5-24. DOI: 10.1111/0952-1895.00121

DUNLOP, Claire. 2017. The irony of epistemic learning: epistemic communities, policy learning and the case of Europe's hormones saga. Policy and Society. v. 36, n. 2, pp. 215-232. DOI: $10.1080 / 14494035.2017 .1322260$

DUNLOP, Claire. 2009. Policy transfer as learning: capturing variation in what decision-makers learn from epistemic communities. Policy Studies. v. 30, n. 3, pp. 289-331. DOI: 10.1080/01442870902863869

FOLI, Rosina; BÉLAND, Daniel; FENWICK, Tracy Beck. 2017. How instrument constituencies shape policy transfer: a case study from Ghana. Policy and Society. v. 35, n. 1, pp. 108-124 DOI: $10.1080 / 14494035.2017 .1377987$

FRANZONI, Juliana Martínez; VOOREND, Koen. 2011. Actors and ideas behind CCTS in Chile, Costa Rica and El Salvador. Global Social Policy. v. 11, n. 2-3, pp. 279-298. Disponível em: https://bit.ly/3iODAkS. Acesso em: 16 ago. 2021.

GARAY, Maria Candelária. 2010. Including the outsiders: social policy expansion in Latin America. Tese de Doutorado em Ciência Política. Berkley: Universidade da Califórnia.

GARCIA, Marito; MOORE, Charity. 2012. The cash dividend: The rise of cash transfer programs in Sub-Saharan Africa. Washington, D.C.: World Bank. Disponível em: https://bit.ly/37NsmXx. Acesso em: 16 ago. 2021.

HAAS, Peter M. 1992. Introduction: epistemic communities and international policy coordination. International Organization. v. 46, n. 1, pp. 1-35. DOI: 10.1017/S0020818300001442 
HAAS, Peter M.; HAAS, Ernest B. 1995. Learning to learn: improving international governance. Global Governance. v. 1, n. 2, pp. 255-284.

HALL, Peter. 1993. Policy paradigms, social learning, and the State: the case of economic policymaking in Britain. Comparative Politics. v. 25, n. 3, pp. 275-296. DOI: 10.2307/422246

HOWLETT, Michael; RAMESH, M.; SAGUIN, Kidjie. 2018. Difusão dos PTCs da América Latina para a Ásia: o caso do programa 4Ps nas Filipinas. Revista de Administração Pública. v. 52, n. 2, pp. 264-284. DOI: $10.1590 / 0034-761220170020$

$\mathrm{KARCH}$, Andrew. 2007. Emerging issues and future directions in state policy diffusion research. State Politics EF Policy Quarterly. v. 7, n. 1, pp. 54-80. Disponível em: https://bit.ly/37NBcod. Acesso em: 16 ago. 2021. KERSTENETZKY, Celia Lessa. 2017. Foi um Pássaro, Foi um Avião? Redistribuição no Brasil no Século XXI. Novos Estudos CEBRAP. v. 36, n. 2, pp. 15-34. DOI: 10.25091/S0101-3300201700020002

KINGDON, John. 1984. Agendas, alternatives, and public policies. Boston: Little, Brown and Company.

LAGOS, Ricardo. 2002. Mensaje al país del Presidente de la Republica. $21 \mathrm{de}$ maio de 2002. Disponível em: https://bit.ly/3g9f1gW. Acesso em:

LANZARA, Arnaldo; BICHIR, Renata. 2019. Not only financialization: recent trends in Brazilian social policies. Development and Change. v. 50, n. 3, pp848-861.

LARRAÑAGA, Osvaldo; CONTRERAS, Dante. 2010. Chile Solidario y el Combate a la Pobreza. In: LARRAÑAGA, Osvaldo; CONTRERAS, Dante. (ed.) Las nuevas políticas de protección social en Chile. Santiago: Uqbar Editores, 2010. pp. 43-76.

LAVINAS, Lena. 2017. The Takeover of Social Policy by Financialization: The Brazilian Paradox. New York: Palgrave Macmillan.

LEITE, Cristiane Kerches da Silva; PERES, Ursula. 2013. Origem e disseminação do Programa Bolsa Família: aproximações analíticas com o caso mexicano. Revista do Serviço Público. v. 64, n. 3, pp. 351-376. DOI: $10.21874 /$ rsp.v64i3.128

LEITE, Cristiane Kerches da Silva; PERES, Ursula Días; BELIX, Laila. 2013. Ideias, contextos e políticas sociais na América Latina: uma análise dos programas de transferência de reda condicionada no Brasil e no México. In: LUKIC, Meline Rocha; TOMAZINI, Carla. (ed.). As ideias também importam: abordagem cognitiva e políticas públicas no Brasil. Curitiba: Juruá, 2013. pp. 145-167. 
LÖBLOVÁ, Olga. 2017. When epistemic communities fail: exploring the mechanism of policy influence. Policy Studies Journal. v. 46, n. 1, pp. 160189. DOI: $10.1111 /$ psj.12213.

MARSH, D.; SHARMAN, J. C. 2010. Policy diffusion and policy transfer. In: EVANS, M. (ed.) New directions in the study of policy transfers. London: Routledge, 2010. pp. 32-51.

MEHTA, Jal. 2011. The varied roles of ideas in politics. In: BÉLAND, Daniel; COX, Robert Henry. (ed.) Ideas and Politics in Social Science Research. Oxônia: Oxford University Press, 2011, pp. 23-46.

MONTEIRO, I. 2011. Integração de políticas sociais: um estudo de caso sobre o Bolsa Família. Dissertação de Mestrado em Bens Culturais e Projetos Sociais. Rio de Janeiro: Fundação Getúlio Vargas.

MULLER, Pierre. 2011. Expliquer le changement: l'analyse cognitive des politiques publiques. In: MULLER, Pierre. Les politiques publiques. 9. ed. Paris: PUF, 2011. pp. 53-86.

OLIVEIRA, Maria Clara. 2018. Ideias e Políticas Públicas. Considerações a partir da análise de programas de transferência monetária na África do Sul, no Brasile no Chile. Tese de Doutorado em Ciência Política. São Paulo: USP.

OLIVEIRA, Maria Clara; OSORIO-GONNET, Cecilia. No prelo. Mudanças e continuidades na passagem do Chile Solidario ao Ingreso Ético Familiar.

OLIVIER DE SARDAN, Jean-Pierre. 2018. Miracle mechanisms, travelling models, and the revenge of contexts. cash transfer programs: a textbook case. In: Olivier de Sardan Jean-Pierre; PICCOLI, Emmanuelle. (ed.). Cash transfers: an anthropological approach. The revenge of contexts. New York: Berghahn Press.

ONG, Aihwa. 2006. Neoliberalism as exception. Mutations on citizenship and sovereignty. Durham: Duke University Press.

OSORIO-GONNET, Cecilia. 2018. ¿Aprendiendo o emulando? Cómo se difunden las politicas sociales en América Latina. Santiago: LOM Ediciones. OSORIO-GONNET, Cecilia. 2015. Mecanismos de difusión de los programas de transferencia condicionada en América Latina. El caso chileno. Íconos - Revista de Ciencias Sociales, n 53, pp. 31-48. DOI: $10.17141 /$ iconos.53.2015.1540

OSORIO-GONNET, Cecilia; Vergara, José Migual. 2019. Programas de transferencia condicionada frente a frente: los casos de Chile, Paraguay e Colombia (2000-2012). DAAPGE - Documentos y Aportes en Administración Pública y Gestión Estatal, v. 19, n. 32, pp. 109-140.

DOI: 10.14409/daapge.v19i32.8483

OSORIO-GONNET, Cecilia; OLIVEIRA, Maria Clara; VERGARA, José Miguel. 2020. International development cooperation as one of the 
triggers for the process of public transfer. Estudos Internacionais., v. 8, n. 2, pp. 8-26. DOI: 10.5752/P.2317-773X.2020v8n2p8-26

PALMA, Julieta; URZÚA, Raúl. 2005. Anti-poverty Policies and Citizenry: the "Chile Solidario" Experience. MOST-2 Policy Papers 12. Paris: UNESCO. Available at: https://bit.ly/3CR7I7k. Acesso em: 30 ago. 2015.

PECK, Jamie, THEODORE, Nik. 2015. Fast Policy: Experimental statecraft and the thresholds of neoliberalism. Minneapolis: University of Minnesota Press. RACZYNSKI, Dagmar. 1994. Social policies in Chile. Origin, transformation, and perspectives. University of Notre Dame, Kellogg Institute for International Studies, Democracy and Social Policies Series, Working Paper \#4. Disponível em: https://bit.ly/2UszTYY. Acesso em: 16 ago. 2016. REINIGER, Taly; CASTRO-SERRANO, Borja; FLOTTS, Marcela; VERGARA, Mónica; FUENTEALBA, Ana. 2016. Conditional cash transfers: social work and eradicating poverty in Chile, International Social Work. v. 61, n. 2, pp. 289-301. DOI: 10.1177/0020872816631601

SABATIER, Paul; JENKINS-SMITH, Hank. 1993. The Advocacy Coalition Framework: Assessment, Revisions and Implications for Scholars and Practitioners. In: SABATIER, Paul; JENKINS-SMITH, Hank. (ed.). Policy Change and Learning: An Advocacy Coalition Approach. Boulder: Westview 244 Press, 1993, pp. 211-35.

SABATIER, Paul A.; WEIBLE, Christopher M. 2007. The Advocacy Coalition Framework: Innovations and Clarifications. In: SABATIER, Paul A. (ed.) Theories of the Policy Process. $1^{\text {st }}$ ed. New York: Routledge, 2007. pp. 189-220. SILVA, Michelle Graciela Morais de Sá e. 2010. Conditional cash transfers and education: united in theory, divorced in policy. Tese de Doutorado apresentada à Graduate School of Arts and Sciences. New York: Columbia University

SILVA, Evelise Zampier; KAUCHAKJE, Samira; DELAZARI, Luciene Stamato. 2017. La política de persuasión del Banco Mundial en sus informes de desarrollo mundial (1990-2015), América Latina Hoy. n. 75, Pp. 75-100. Disponível em: https:/ /bit.ly/3sdHIUd. Acesso em: 13 ago. 2021.

SIMONS, Arno; Voß, Jan-Peter. 2018. "The concept of instrument constituencies: accounting for dynamics and practices of knowing governance”. Policy and Society. v. 37, n. 1, pp. 14-35. DOI: $10.1080 / 14494035.2017 .1375248$.

STONE, Diane. 2012. Transfer and translation policy. Policy Studies. v. 33, n. 6, pp. 483-499. DOI: 10.1080/01442872.2012.695933

STONE, Diane. 2004. Transfer agents and global networks in the "transnationalization" of policy. Journal of European Public Policy. v. 11, n. 3, pp. 545-566. DOI: 10.1080/13501760410001694291 
SUGIYAMA, Natasha Borges. 2011. The diffusion of conditional cash transfer programs in the Americas. Global Social Policy. v. 11, n. 2-3, pp. 250-278. DOI: 10.1177/1468018111421295

SUGIYAMA, Natasha Borges. 2008a. Theories of policy diffusion: social sector reform in Brazil. Comparative Political Studies. v. 41, n. 2, pp. 193216. DOI: 10.1177/0010414007300916

SUGIYAMA, Natasha Borges. 2008b. Ideology and networks. The politics of social policy diffusion in Brazil. LARR. v. 43, n. 3, pp. 82-108. Disponível em: https://bit.ly/3iQgLxv. Acesso em: 13 ago. 2021.

TOMAZINI, C. 2010. As coalizões de causa em torno das políticas de transferência condicionadas: olhar cruzado Brasil-México. Carta Internacional. v. 5, n 2, pp. 37-55. Disponível em: https://bit.ly/2XrXHxd. Acesso em: 13 ago. 2021.

VERAS SOARES, Fabio. 2012. Oportunidades and Bolsa Família: a comparative perspective on their evaluation. IPC-IG One Paper $\mathrm{n}^{\circ} 177$.

VOß, Jan-Peter; SIMONS, Arno. 2014. Instrument constituencies and the supply side of policy innovation: the social life of emissions trading. Journal of Environmental Politics, v. 23, n. 5, pp. 735-754. Disponível em: https://bit.ly/37IATLi. Acesso em: 13 ago. 2021. 
Transferência monetária no Brasil e no Chile 


\section{TRANSFERÊNCIA MONETÁRIA NO BRASIL E NO CHILE: COMPARANDO IDEIAS E O PAPEL DE INSTRUMENT CONSTITUENCIES}

MARIA CLARA OLIVEIRA

RENATA MIRANDOLA BICHIR

Resumo: Este artigo discute a adoção na esfera nacional de políticas disseminadas globalmente. $\mathrm{O}$ artigo demonstra a vantagem de se recorrer aos conceitos de comunidade epistêmica e de instrument constituency para examinar não só a disseminação de políticas públicas, mas também analisar a inserção de problemas na agenda política nacional, a discussão de soluções possíveis e a formulação de políticas públicas. Tomamos como caso empírico as políticas de transferência monetária adotadas no Brasil e no Chile e analisamos, de modo comparativo, as principais ideias que fundamentam sua adoção, discutindo como elas informam os desenhos dos programas. Os resultados apontam a existência de comunidades epistêmicas e de instrument constituencies, que atuam no plano local de distintos modos e que se revelam nos dois casos-chave na adoção de políticas de transferência monetária.

Palavras-chave: Ideias; Comunidades Epistêmicas; Instrument Constituency; Transferência Monetária; Brasil; Chile.

\section{CASH TRANSFERS IN BRAZIL AND IN CHILE: COMPARING IDEAS AND THE ROLE OF INSTRUMENT CONSTITUENCIES}

Abstract: This article discusses the adoption at the national level of public policies that were disseminated at the global level. The article shows that the concepts of epistemic community and instrument constituency are useful not only in the analysis of policy diffusion, but also to look at the inclusion of certain topics in the political agenda, the debates regarding the solution to these problems and 
policy design processes. We examine cash transfer programs in Brazil and Chile and compare the main ideas behind their adoption and discuss how they have informed the design of the two programs. Our results point to the existence of epistemic communities and instrument constituencies, who play a key role in the adoption of cash transfers in both countries and who act differently according to the context.

Keywords: Ideas; Epistemic Community; Instrument Constituency; Cash Transfers; Brazil; Chile.

Recebido:03/10/2019 Aprovado: 26/07/2021 